\title{
Exhaust (broadband) noise simulation of a realistic turbofan forced mixer by using a CFD/CAA-approach.
}

\author{
Paul Traub ${ }^{1}$ and Dominik Broszat. ${ }^{2}$ \\ MTU Aero Engines, Munich, 80995, Germany \\ Thomas Röber ${ }^{3}$ \\ German Aerospace Center, Cologne, 51147, Germany and \\ Jens Wellner ${ }^{4}$ \\ German Aerospace Center, Cologne, 51147, Germany
}

The growing demand of low noise civil aircraft brings out technical challenges which require the application of computational aeroacoustics (CAA) methods within the development process of jet engines. This paper focuses on the simulation of exhaust jet noise based on a realistic turbofan lobe-forced mixer configuration and operating condition. The present study aims at showing the ability of a Ffowcs Williams-Hawkings (FWH) approach for solving the acoustical sources of a complex turbulent (sheared) flow and for predicting the far field (broadband) noise based on a Detached Eddy Simulation (DES). The evaluation of the prediction results by comparing with benchmark data yield some general guidelines given for model improvements.

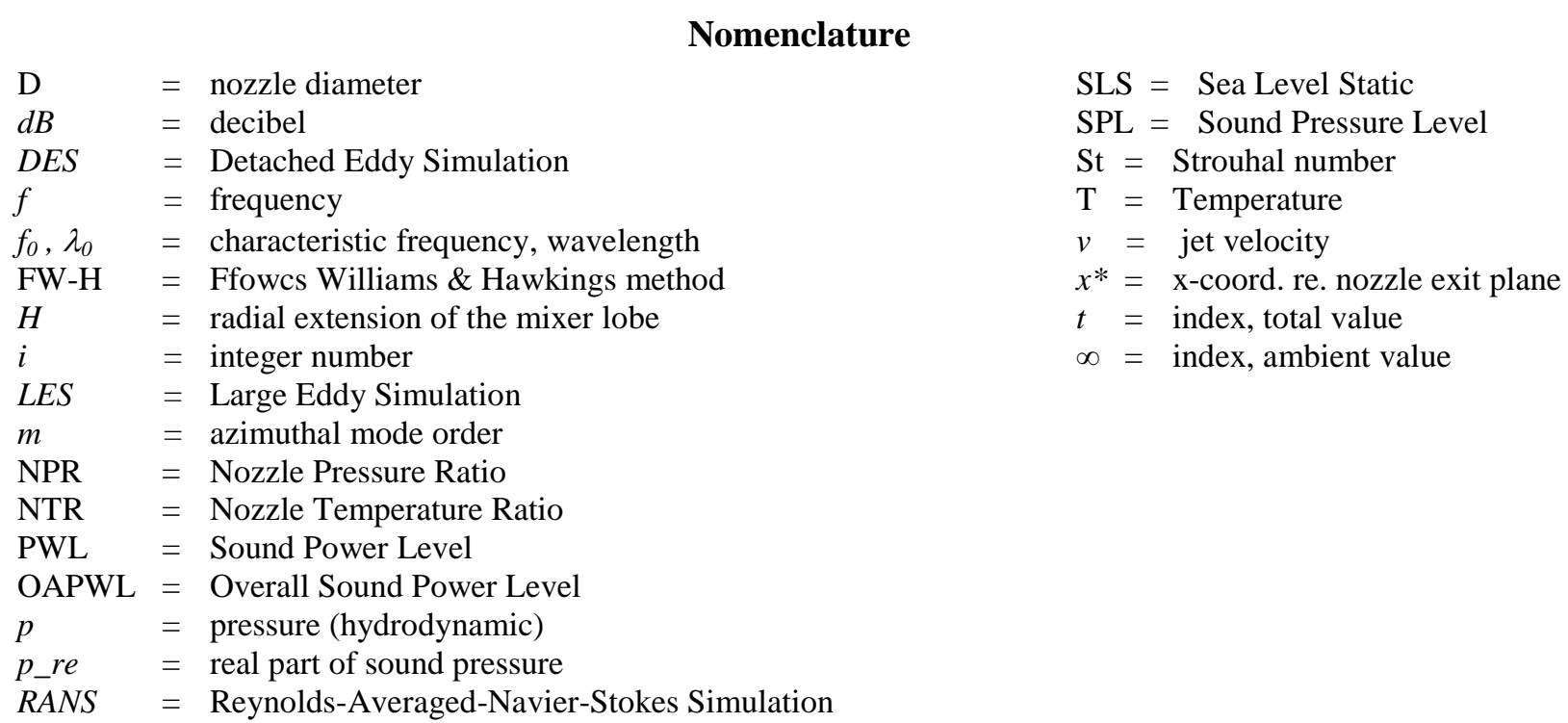

\footnotetext{
${ }^{1}$ Diplom Phycisist, Dept. Aerodynamics, Aeroelasticity, Aeroacoustics Turbine (TEAT), P.O.Box 5006 40, 80976 Munich, Germany, AIAA Member.

${ }^{2} \mathrm{PhD}$, Dept. Aerodynamics, Aeroelasticity, Aeroacoustics Turbine (TEAT), P.O.Box 5006 40, 80976 Munich, Germany, AIAA Senior Member.

${ }^{3}$ Now: Curator, Power Machinery, Deutsches Museum, Museumsinsel 1, 80538 Munich, Germany, AIAA Member.

${ }^{4}$ Research Engineer, German Aerospace Center,Institute of Propulsion Technology, Linder Hoehe,51147 Cologne, Germany, AIAA Member.
} 


\section{Introduction}

$\mathrm{J}$

et noise from turbofan engines is one of the major contributors to aircraft noise in particular during take-off.

Therefore, modern small-to-medium turbofan engines of bypass ratio 5 to 6 feature an exhaust mixer, where the bypass (cold) air is mixed with the core (hot) exhaust gases, before leaving a common nozzle to the environment. The typical mixer design as considered here forces the two streams to mix by lobed and intertwined chutes in order to increase the mixing efficiency which reduces disproportionately the core stream velocity and thus the broadband (exhaust) noise radiated to the far field. The application of computational methods like CFD and CAA approaches becomes more important in the development process of jet engines in order to improve the complex shape of the mixer design on the one side and to increase the accuracy of the exhaust noise prediction on the other side.

\section{Methodology / Numerical Methods}

\section{A. Flow Simulation / Computational Setup}

It is a challenging task for numerical schemes to capture the turbulent mixing flow and acoustic field for realistic boundary conditions. The used approach, simulating the flow field for this study is an adaptation of a classical DES to two equation models [Ref. 1]. The CFD results have been provided by DLR (German Aerospace Center) by using the DLR flow solver TRACE applied to a full scale mixer (segment) model provided by MTU Aero Engines. TRACE solves the Reynolds-averaged Navier-Stokes equations on hybrid structured/unstructured multi-block meshes using a finite-volume method. For time integration, a higher order implicit time-stepping method is applied [Ref. 2].

The computational setup is described in more detail in the following. In addition, an overview is given of the global grid features and criteria for the mesh refinement. Fig. 1 shows the geometry of the selected lobed mixer containing 14 segments. DES computations are performed for one mixer segment within the azimuthal sector of 360/14 deg. To assure the feasibility of DES on the angular sector, the pylon is not taken into account in the computation. Jet flow parameters are specified for a realistic sea level static (SLS) operating condition. The operating condition is defined by the pressure ratio (NPR) and temperature ratio (NTR) as given in Tab. 1 for the primary and secondary nozzle. In this work, the nozzle pressure and temperature ratios are defined as NPR=p/ $/ \mathrm{p}_{\infty}$ and NTR $=T_{t} / T_{\infty}$, where the subscripts $t$ and $\infty$ refers to total and ambient properties, respectively. The penetration height $(\mathrm{H} / \mathrm{D})$ of the considered mixer configuration defined as the difference between the maximum and minimum radii at the end of the mixer referred to the nozzle diameter is also given in Tab. 1 for a better comparability with other studies. The modeled sector of the jet configuration is outlined in figures 2, 3 and 4, partly including the FW-H surface(s) used to compute the far-field sound (see section II.B).

In order to estimate the relevant frequency range from the jet noise spectrum, the jet noise sound power spectrum has been predicted by using an in-house semi-empirical calculation method. Deducing from that, a characteristic frequency $f_{0}$ of $1000 \mathrm{~Hz}$ has been defined. Below that frequency the most spectral content of sound power is expected. This information is mandatory to define the time step and the grid refinement for the DES computations. The results in the time domain for a specified time interval contain the information on the turbulent structures. During the generation of the CFD mesh, care has to be taken on the mesh refinement to resolve the acoustic waves temporally and spatially to ensure propagation in any direction. This requirement must be met independently of the local flow conditions. Furthermore, the nature of the filter in the DES scheme favours isotropic meshes, i.e. shifting from RANS to LES style modelling is based on the maximum cell dimension, so that refinements in just one direction are superfluous in regions where the model is expected to run in LES mode. Therefore, the mesh was built in such a way, that, for the fluid at rest, at least ca. 80 nodes per wave length $\lambda_{0}$ of a pressure wave at the characteristic frequency $f_{0}$ for free-field conditions were available. In the worst case of an upstream travelling wave in the bypass, more than 40 nodes per wave length were still retained. The mesh was considerably refined in wallnormal direction in the boundary layers, where the turbulence model can be expected to run in RANS mode. Thus, in wall-normal direction, care was taken to ensure $\mathrm{y}^{+} \approx 5$ for all solid boundaries, while in axial and lateral directions, the above mentioned constraints (at least 40 nodes per wave length) were kept. In order to ensure that a correct boundary layer velocity profile was enforced in spite of the first off-the-wall node being located above the viscous sub-layer, a continuously formulated wall function was used. The turbulence model boundary conditions were modified correspondingly to allow a smooth transition between sub-layer and log-layer profiles. In the shear layers, the mesh refinement that emanates from the boundary layers is retained in order to ensure a good resolution of mean flow gradients in regions where the model might run in RANS mode. Furthermore, the mesh has been 
refined in axial and lateral directions by means of three unstructured block-cuts downstream of the mixer lobes to allow the DES model to switch to LES mode in this region. The mesh extends axially by 2.5 nozzle radii downstream from the nozzle exit plane. The planar extension related to the centerline amounts 2.5 nozzle radii. In total, the computational mesh contains approximately 40 million nodes for the one segment of the mixer model.

Fig. 2 exemplifies the global structure of the unsteady flow, including the shear layers between core and bypass and between nozzle and exterior flow. The iso-vorticity surface for $f=2 \mathrm{kHz}$ has been coloured with the air temperature, from blue (cold) to red (warm) to illustrate the mixing between core and bypass flow. Behind the mixer lobes, a complex shear layer pattern emerges with turbulent structures becoming larger with downstream convection far apart from the mixer. As shown in Fig. 3, there are flow regions with attached flow, which could be captured by the RANS-method, e.g. the duct casing upstream of the mixer or nozzle. On the other hand, there are regions behind the mixer segment with prevalent turbulent structures, which have to be resolved by the mesh and calculated by the LES model.

Regarding the digital signal processing of the CFD data, one period (1/1000 s) of frequency $f_{0}$ was resolved with 512 time steps, 32 of which were recorded for the acoustic analysis. Subject to the Nyquist theorem, a limit of 16 $\mathrm{kHz}$ i.e. the half of the sampling rate defines in the considered case the analyzable frequency range. A frequency resolution of $50 \mathrm{~Hz}$ results from the finite simulation time of the time signal. The computation was initialized from a steady-state RANS computation. Before data recording started, a sufficient number of periods, approximately 75 periods $(0.075 \mathrm{~s})$, had to be computed to allow for unsteady structures to develop in the shear layers. In total, the 3-D field data were recorded for the last 16 periods.

The high spatial resolution of the DES requires a large amount of disk space to save the entire 3-D flow field over a time period sufficient for the acoustic analysis. To accelerate the acoustic analysis, a pre-processing tool has been used to map the unsteady quantities of the acoustic source terms on the topology of a defined analysis surface in order to apply the Ffowcs Williams \& Hawkings (FW-H) method [Ref. 3] for far field noise simulation. The 2-D database was recorded for the last 20 periods.

\section{B. Far-Field Sound Simulation / Computational Setup}

For computing the far field acoustic fluctuations, the commercial FW-H solver ACTRAN [Ref. 4] has been used. The solver is based on an advanced integration technique of the FW-H equation which can be seen as an extension of the Lighthill acoustic analogy. The FW-H utility takes as an input frequency or time domain information on a user-defined surface. Some criteria have been taken into account for the surface specification. The FW-H surface represents the interface between an inside domain supporting an unsteady flow and an outside domain at rest or supporting a uniform flow. Further on, all acoustic sources must be enclosed and all the mean flow quantities on and across the surface must be uniform. In theory, the surface has to enclose the unsteady domain completely, but for jet noise evaluation, some authors [e.g. Ref. 5] have demonstrated that it is sufficient to use open surfaces without loss of accuracy.

The present study deals with CFD-data which have been processed for 3 predefined FW-H surfaces, as shown in Fig. 4 marked in green, yellow and blue. For computing the sound radiation to the far-field, i.e. the sound pressure directivity in decibel scale (SPL), microphone positions on a polar arc are defined for a radial distance of $46 \mathrm{~m}(\approx$ $150 \mathrm{ft}$, typically used for static engine measurements) at angles ranging from $40 \mathrm{deg}$ to $140 \mathrm{deg}$. The center point is located on the centerline $(y=z=0)$ at $x$-position of the nozzle exit plane. The green marked surface extends at a larger radius parallel to the reference (yellow) surface. The idea behind this is to study the effect of different radial positions on the SPL directivity. The contour and the radial distance of the 'open' surfaces (yellow, green) relative to the nozzle exit have been chosen in such a way that they are preferably outside the unsteady flow field (Fig. 3), as required by the classical theory of FW-H. In contrast to that, the blue marked control surface follows for the most part the contour of the reference face in axial direction, but the radial extended part of the surface intersects with the fully turbulent flow. This case does not comply with the above mentioned criterion and should clarify the influence on the FW-H solution.

The ACTRAN code features the functionality of a periodic boundary condition, i.e. the effort is limited to model the angular sector instead of the complete (full domain) configuration. Thus, the computation time and memory demand can be reduced efficiently, provided that the mixer has a periodic shape in the azimuthal direction. Some aspects concerning the feasibility of LES on an angular sector with periodic boundary conditions are described in [Ref. 6]. Then, by using the periodic boundary condition, the sound pressure on a defined field point or microphone position is the sum (complex number) of the contributions of the different reproduced surfaces. The radiated sound 
power of the segment is multiplied by the geometric periodicity in order to estimate the sound power of the full domain model.

For far field analysis, i.e. for the computation of the sound pressure at defined microphone positions in the far field, ACTRAN solves the FW-H equations in the frequency domain by applying the Direct Fourier Transformation (DFT). In general the recorded time samples correspond to a finite or truncated part of a signal provided by CFD simulations. Therefore, the Fourier transformed truncated waveform may result different spectral characteristics compared to a continuous-time signal. To minimize this (leakage) effect, an appropriate window function can be applied. This will enforce a continuity at the end points of the signal and therefore result in a continuous waveform without sharp transitions. Different types of window functions are implemented in ACTRAN; each with their own advantage and preferred application. So for selecting a window function it is advisable to estimate at first the frequency information content of the signal. In principle, the nature of jet mixing creates turbulent eddies which cause a prevalent random sound signal and accordingly a broadband characteristic in the frequency domain. On the other hand, the presence of the mixer adds complexity to the jet flow fields. The nature of the turbulent flow field close to the nozzle exit plane, for example quantified by the turbulent kinetic energy (TKE), shows typically a circumferential periodical pattern, evidently caused by the lobed mixer annulus. This may cause sound modes at frequencies which correlate to the number of lobes. Furthermore, based on practical experience, the forced mixer generates high frequency source noise, commonly labeled "mixer excess noise". The mixer excess noise is most probably associated with a marked increase in turbulence level in the outer shear layer as penetration increases [Ref. 7].

Following the basic principles of signal analysis, the 'rectangular windowing' yields the same energy content for the Fourier solution as for the time signal (amplitude accuracy). It is also self-evident to apply rectangular windowing, equivalent to uniform windowing, if the type of signal is random. For comparison, FW-H far-field predictions have also been performed using the Hanning window, which is advised for a better frequency resolution to discover potential tonal spectral characteristics as e.g.'mixer excess noise'. But in contrast to the rectangular window, the Hanning window reduces the energy content of the original signal. As outlined more detailed in chapter III, the prediction results based on both window functions, 'rectangular' and Hanning, have been compared with benchmark data.

\section{Results/ Evaluation}

\section{A. Preliminary Assessment}

Before starting the computations relevant for the benchmark test, Actran results have been successfully crosschecked with the outcome of DLRs FW-H method as described in [Ref. 1]. Within previous full domain simulations i.e. applying the periodic boundary conditions, the sound power spectra (PWL) based on the CFD-data of the given 3 various FW-H surfaces have been predicted by using rectangular windowing (Fig. 5). In view of the benchmark test as described in the next chapter, it is worth noting, that the surfaces (maximum axial extension one nozzle diameter downstream of nozzle exit plane: $\mathrm{x} / \mathrm{D}=1$ ) cover not only acoustic sources relevant for forced mixer noise but also sources influenced by the shearing action caused by the relative speed between the exhaust jet and the atmosphere. Regarding Fig. 5, the PWL spectra follow an overall trend as typical for jet noise. Thus, the maximum level occurs in the low frequency range, around the $1 / 3$ octave mid frequency $315 \mathrm{~Hz}$. The total sound power (OAPWL) for the "open" surfaces 05000 and 05100 are almost the same. Deducing from that, both surfaces - even type 05000 radially closer to the unsteady flow field - are suitable to meet the FWH-interface criterion. Compared with the "radially closed" surface 04999, the corresponding OAPWL is clearly higher (about $6 \mathrm{~dB}$ ), because the surface enclose even more acoustic sources. Although the surface topology does not comply with the above mentioned interface criterion, the shape of the spectrum follows the global trend.

\section{B. Benchmarking}

This study focus on benchmarking the prediction results with obtained mixer noise data, generated by a further developed version of the semi-empirical ISVR/Purdue method [Ref. 7, 8,9], which is extensively validated with scale model lobed mixer data and full-scale engine noise data, as well. The basis of the semi-empirical method relies on the fact, that the jet plume can be devided axially into regions downstream from the nozzle exit plane. The jet noise sources are then characterized by the jet parameters velocity, temperature, diameter and turbulent properties of 
the corresponding regions. For the purpose of comparability, the FW-H solution has been post-processed not only for the whole surface, but also for subareas enclosing the turbulent sources at various relative axial lengths $\mathrm{x} * / \mathrm{D}$, ranged from $18 \%$ to $100 \%$. Fig. $6 \mathrm{a}$ and $6 \mathrm{~b}$ show the predicted PWL spectra referring to surface 05000 (reference). The comparison of rectangular versus Hanning windowing results shows spectra of similar trends for $\mathrm{x} / \mathrm{D}=18 \%$ and 35\%, characterized by significant peak levels at frequencies $500 \mathrm{~Hz}$ and $6.3 \mathrm{kHz}$. With increasing axial distance downstream from the nozzle exit, the portion of the low frequency broadband noise rises obviously and overshadows the peak level at $500 \mathrm{~Hz}$. In contrast to the result based on Rectangular windowing, the applied Hanning window resolves the peak level at $6.3 \mathrm{kHz}$ even for the whole available FW-H analysing domain. A more detailed investigation by means of the narrow band spectrum (Fig. 7) and azimuthal directivity at nozzle exit plane of the real parts of the sound pressure p_re (Fig. 8) leads to the following finding:

The maximum power level occurs also at the narrow band frequency of $500 \mathrm{~Hz}$ and the level decreases clearly at the neighbouring frequencies. The peak levels at $6.15,6.2,6.6$ and $6.65 \mathrm{kHz}$ contribute significantly to the $1 / 3$ octave level at $6.3 \mathrm{kHz}$. According to Fig. 8, the real part of the sound pressure of the relevant low frequencies are constant across the whole azimuthal range. In contrast to that, the circumferential p_re patterns referring to peak levels in the $1 / 3$ octave band $6.3 \mathrm{kHz}$ are obviously periodic of mode order 14 equivalent to the mixer's lobe number. It has been proven, that the directivity curves in the range below the $1 / 3$ octave frequency of $4 \mathrm{kHz}$ show marginal periodical or none periodical patterns of order $m=14$. The low frequency peak level at $500 \mathrm{~Hz}$ appears to correlate with a Strouhal-number $\mathrm{St}=2$ as given through the relation $\mathrm{St}=\mathrm{f} \cdot \mathrm{D} / \mathrm{v}$, where $\mathrm{v}$ and $\mathrm{D}$ are the jet characteristic velocity and diameter, respectively. The value is within the range of experience $(\mathrm{St}=1$. to 2.5$)$ which have been inferred from noise data of 12-lobed mixer configurations [Ref. 7]. Regarding the interpretation of the characteristic $1 / 3$ octave band level at $6.3 \mathrm{kHz}$, two points of view are self-evident:

On the one hand, the spectral shape deals with high frequency source noise, generated by the forced mixer. On the other hand, the tendency may be probably caused by the periodicity condition from the CFD computation, which distributes the sound energy on sound modes of azimuthal order $\mathrm{m}=\mathrm{i} \cdot 14$, with $\mathrm{i}=0,1$, 2 , etc.. The latter interpretation follows the findings given in [Ref. 6], which compares the results of "classical" full domain with quarter domain simulations by applying periodic boundary condition. These aspects have been taken into consideration by interpreting the prediction results compared with the benchmark data (free-field data i.e. lossless corrected in the sense of atmospheric damping) as shown in Fig. 6b. Thus, the prediction overestimates the mixer noise obviously in the low frequency range around $500 \mathrm{~Hz}$. Regarding the spectral shape around $6.3 \mathrm{kHz}$, the benchmark curve is typically characterized by broadband noise in contrast to the prediction as mentioned above. For the case of the remaining range within $1 \mathrm{kHz}$ and $4 \mathrm{kHz}$, the predicted levels are close to the benchmark curve. But even for that range of frequency, we would expect lower levels compared with the benchmark curve, because the benchmark data are deduced from a wider axially extended region of the jet plume, 5-times magnified as for the computational setup. The observed tendency of global overestimation is in line with the conclusion from [Ref. 6].

\section{Conclusion}

The comparison with benchmark data have demonstrated, that the prediction by using the FW-H surface integral method applied for DES-data, reproduces the main features of the forced mixer noise spectrum and directivity. Thus, the predicted noise is predominant at low frequencies being verified with Strouhal-numbers of characteristic jet parameters. However, concerns are advisable on the interpretation of the absolute levels and especially spectral characteristics linked to azimuthal directivities, showing mode orders of integer multiples of the lobe numbers of the forced mixer. Thus, the usage of DES data based on segment modeling applying periodic boundary conditions modifies turbulent structures artificially which has a strong influence on the acoustic radiation. Therefore, further studies will focus on the comparison of segment domain with classical full domain simulations to improve the degree of accuracy. 


\section{Acknowledgments}

This work has been carried out in the framework of the German Aeronautical Research Program (LuFo) within the joint research project MASSIF-EffekT (Massnahmen zur Schallpegelsenkung im Flugverkehr - Effektive Triebwerkslaermreduzierung $=$ Measure for aircraft noise reduction - effective engine noise reduction). The support of the German Federal Ministry of Economics and Technologies (BMWi) is gratefully acknowledged. The authors would like to thank Christian Weckmüller (DLR, Berlin, Germany) in the context of cross-checking the prepredicted FW-H results and helpful discussions. The authors also greatfully acknowledge Sid-Ali Meslioui and LiJen Chen from Pratt \&Whitney Canada for providing the benchmark data. The help of Yves Detandt and the support-team of Free Field Technologies (FFT, MSC Software Corporation) are gratefully acknowledged.

\section{References}

${ }^{1}$ Wellner, J., Röber, T., Traub, P., “On the development and application of a hybrid approach to the simulation of broadband noise in a lobed mixer", AIAA 2011-2799.

${ }^{2}$ Ashcroft, G., Frey, C., Heitkamp, K., Weckmüller, C., “Advanced numerical methods for the prediction of tonal noise in turbomachinery, Part I: Implicit Runge-Kutta schemes”, ASME Turbo Expo GT2012-69447.

${ }^{3}$ Ffowcs Williams, J.E. \& Hawkings, D.L., "Sound generation by turbulence and surfaces in arbitrary motion", Phil. Trans. Roy. Soc. London, 1151, Ser. A, 321-342, 1969.

${ }^{4}$ ACTRAN, CAE Software Package, Vers. 13.0, Free Field Technologies (MSC Software Corporation), Axis Park Louvain-laNeuve 9, B-1435 Mont-Saint-Guibert, Belgium.

${ }^{5}$ Uzun, A., Lyrintzisi, A.S., Blaisdell G.A., "Coupling of integral acoustics methods with LES for jet noise prediction”, AIAA 2004-0517.

${ }^{6}$ Bodard, G., Bailly, C., "Feasibility of Large-Eddy Simulation on angular sector to evaluate chevron effects on jet noise", AIAA 2010-3846.

${ }^{7}$ Tester, B.J., Fisher, M.J., "A contribution to the understanding and prediction of jet noise generation in forced mixers", AIAA 2004-2897.

${ }^{8}$ Tester, B.J., Fisher, M.J., "A contribution to the understanding and prediction of jet noise generation in forced mixers, Part II: Flight effects", AIAA 2004-2897.

${ }^{9}$ Garrison, L.A., Dalton, W.N. , Lyrintzis A. S., Blaisdell, G. A., "On the development of semi-empirical noise models for the prediction of the noise from jets with forced mixers", AIAA 2004-2898. 
Figures \& Tables
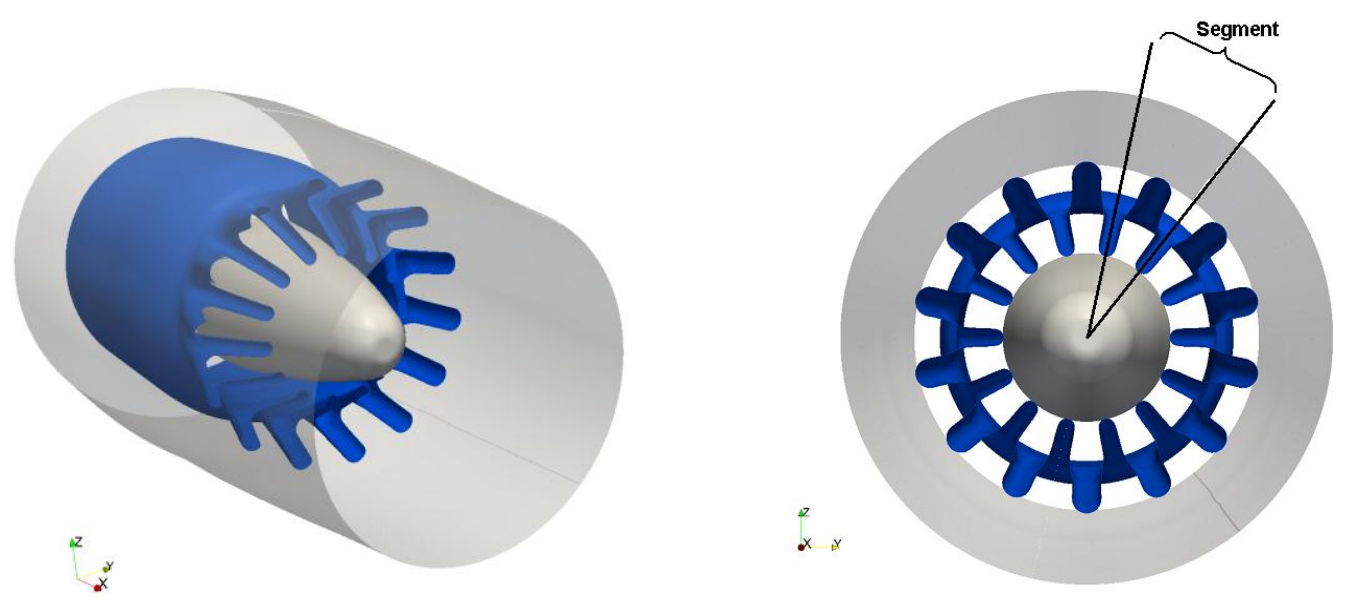

Figure 1. Geometry of the studied forced mixer configuration

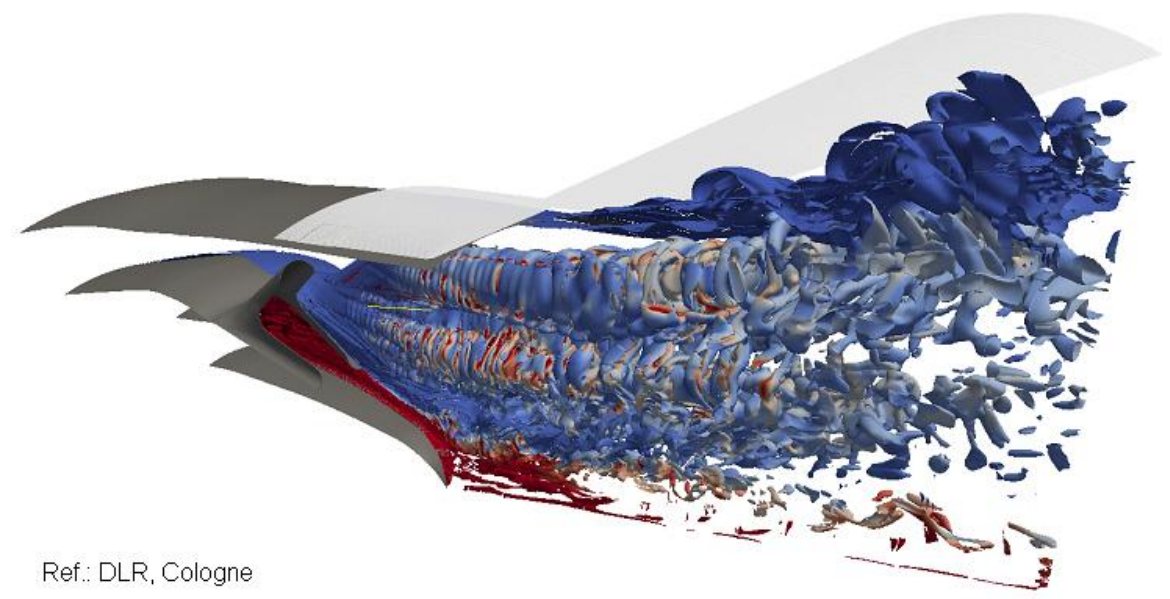

Figure 2. Iso-vorticity surfaces and one exemplary permeable FW-H surface (light gray). 


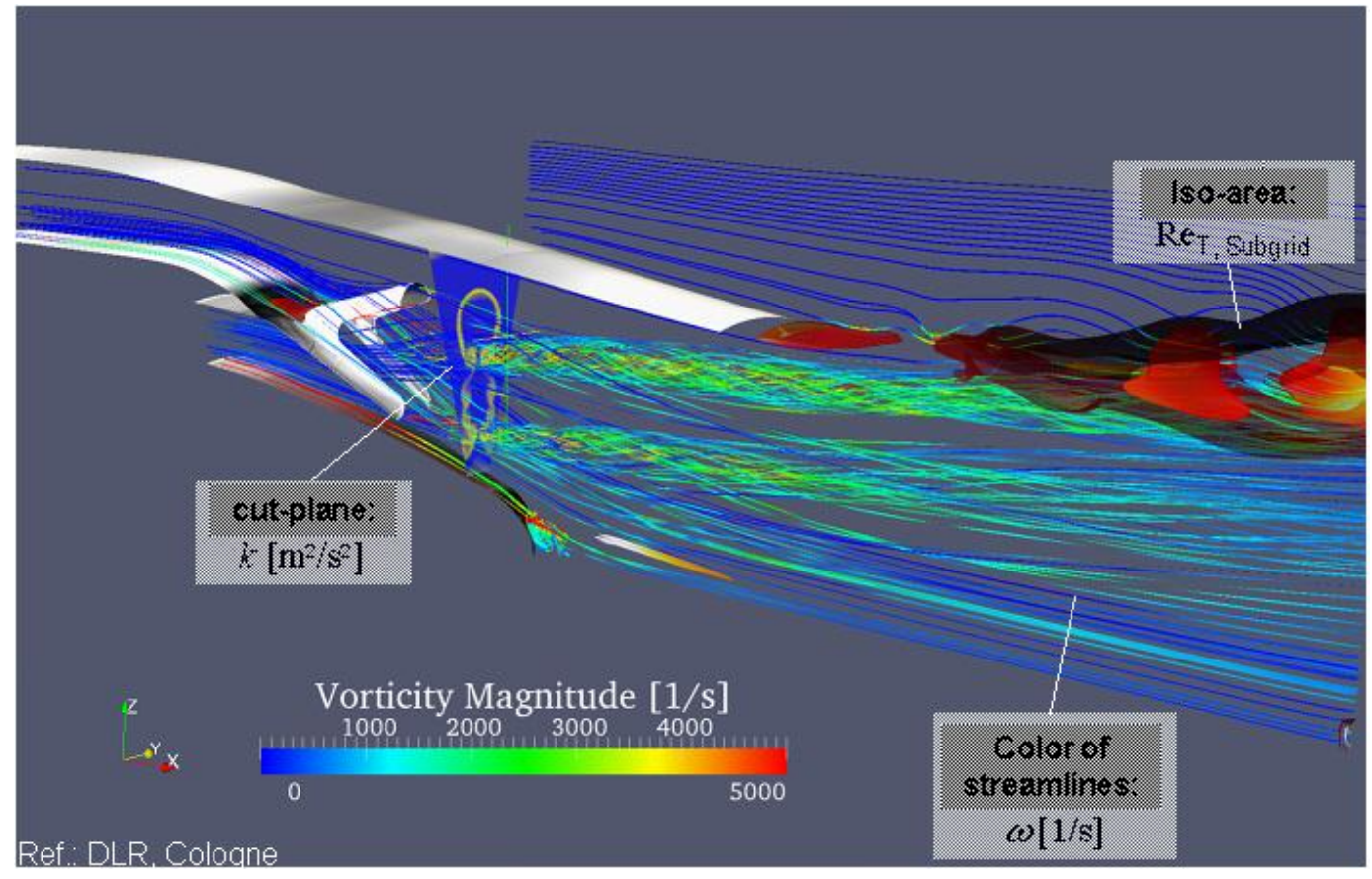

Figure 3. Mixing flow simulation: Vorticity $\omega$ and turbulent kinetic energy $\left(k, R_{T}\right)$. 


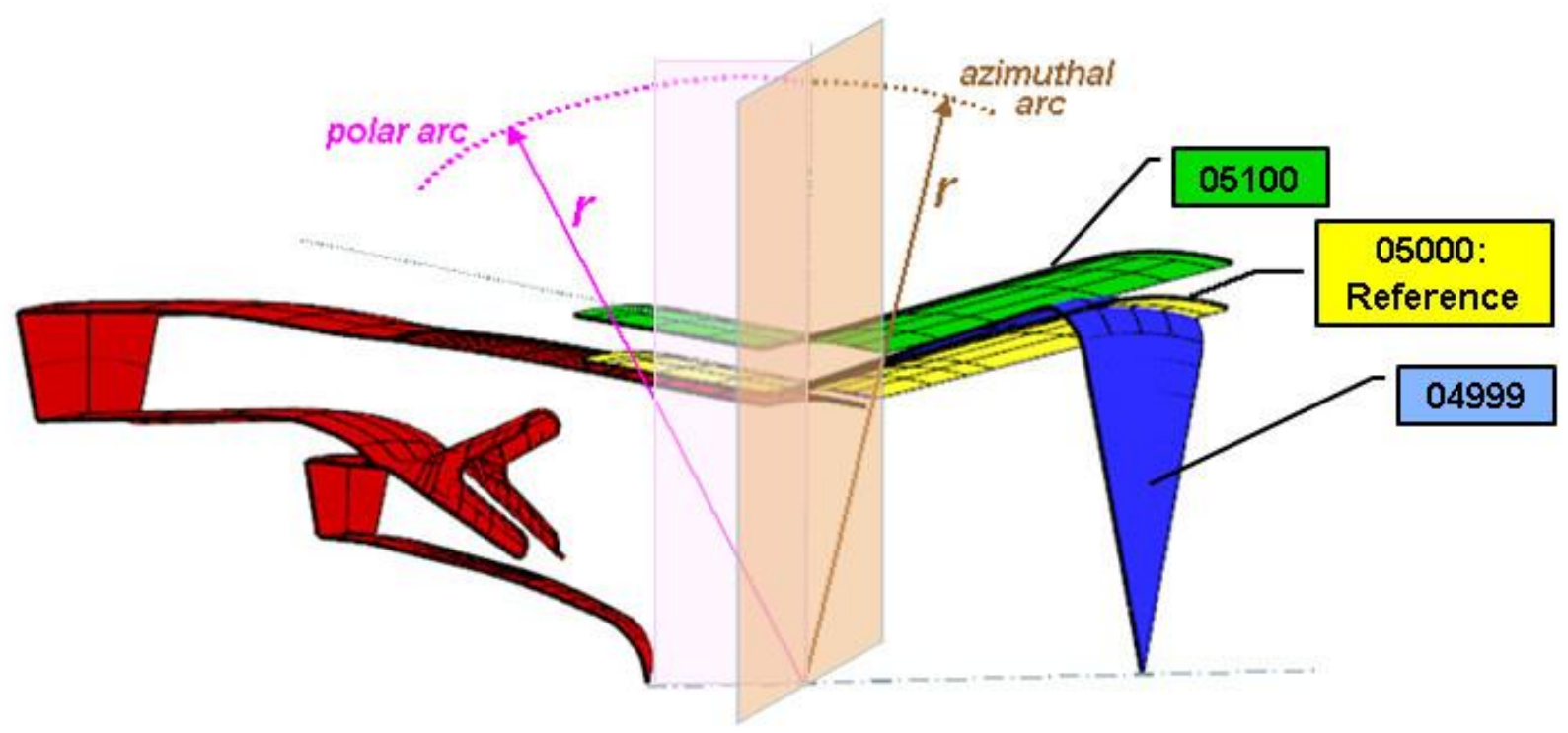

Figure 4. Nozzle-mixer segment (red) with 3 FW-H surfaces (yellow, green, blue).

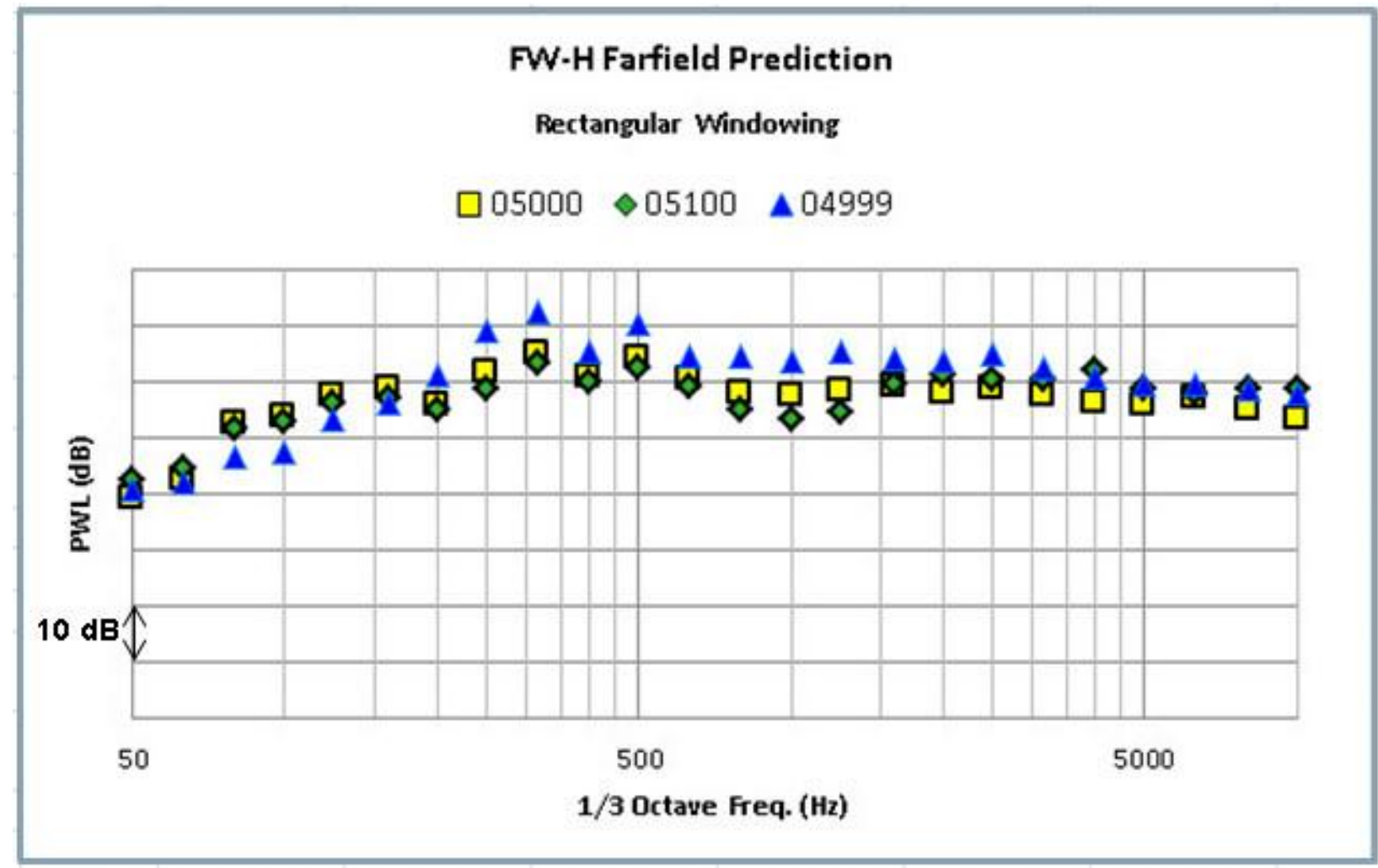

Figure 5. Predicted sound power spectra related to various FW-H surfaces.

American Institute of Aeronautics and Astronautics 


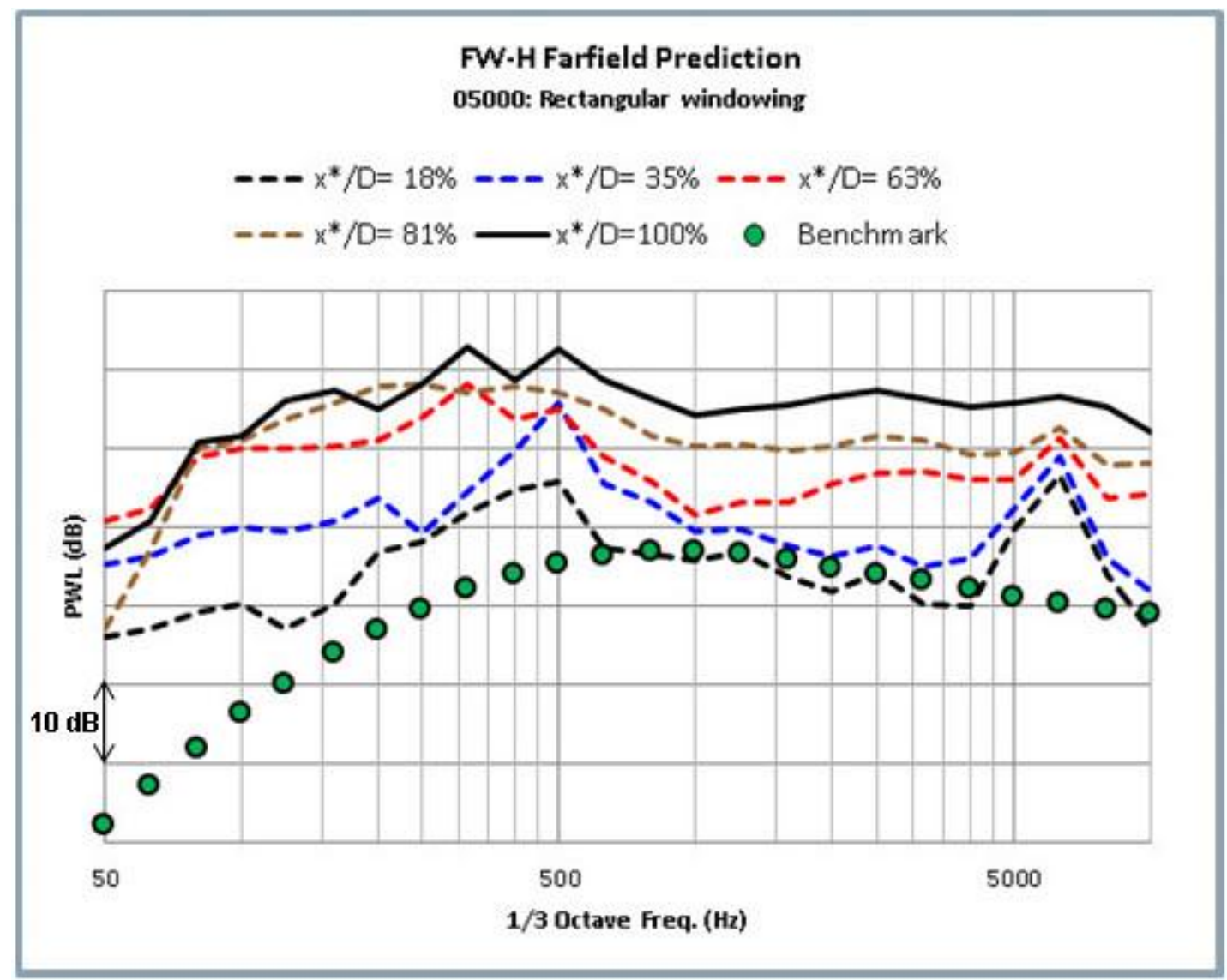

Figure 6a. Benchmark of predicted sound power spectra: Rectangular windowing. 


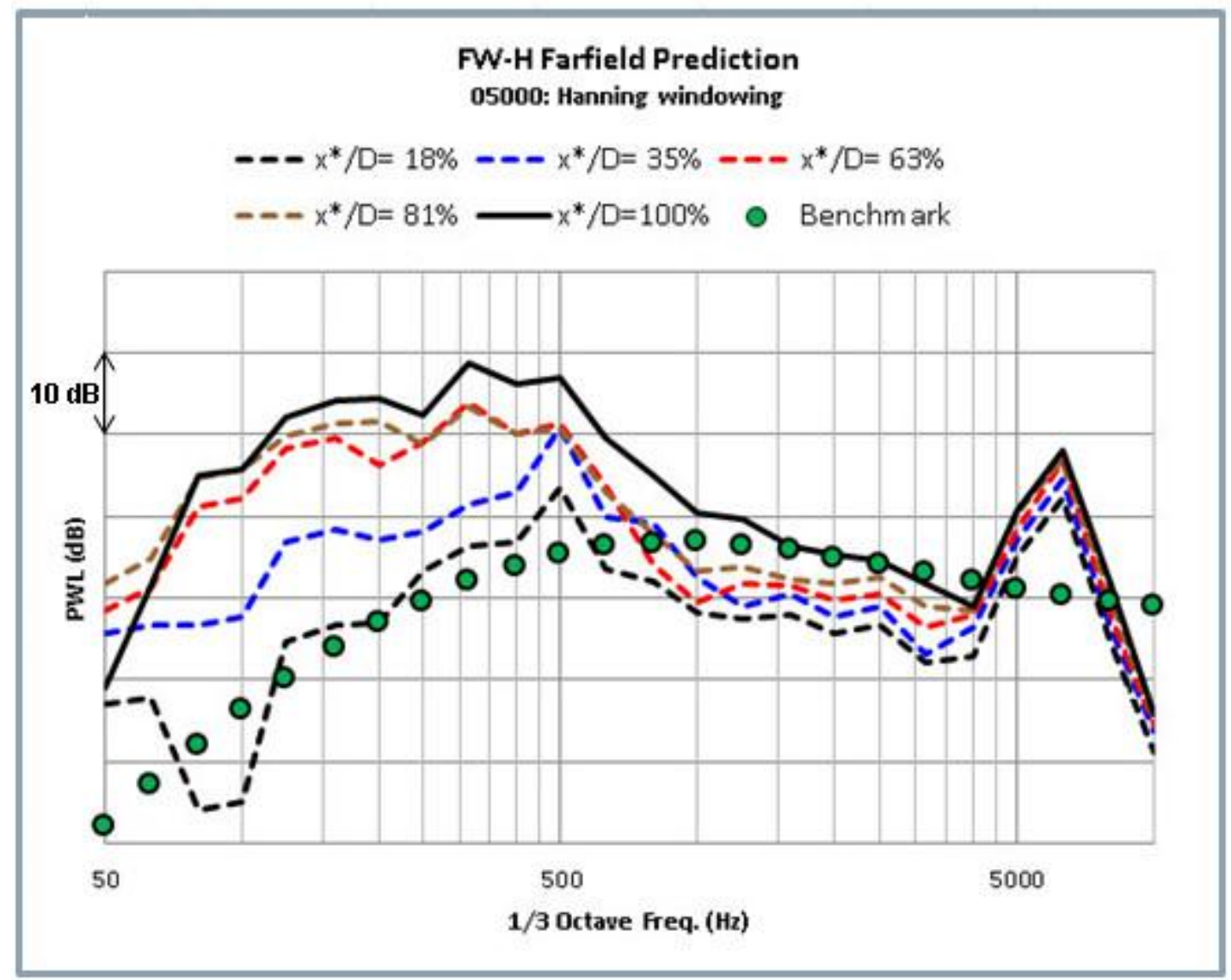

Figure 6b. Benchmark of predicted sound power spectra: Hanning windowing. 


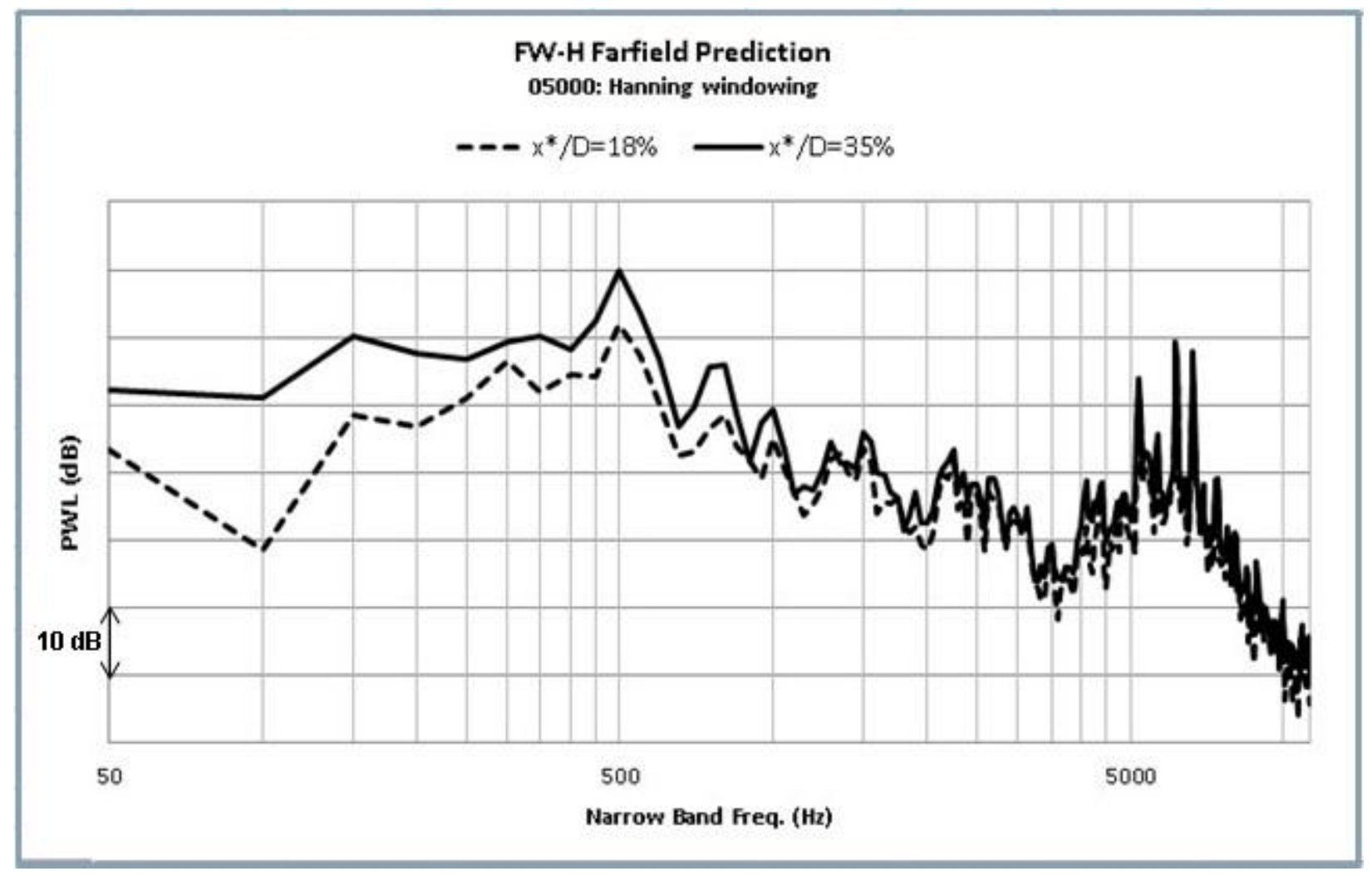

Figure 7. Narrow band sound power spectra: Hanning windowing. 


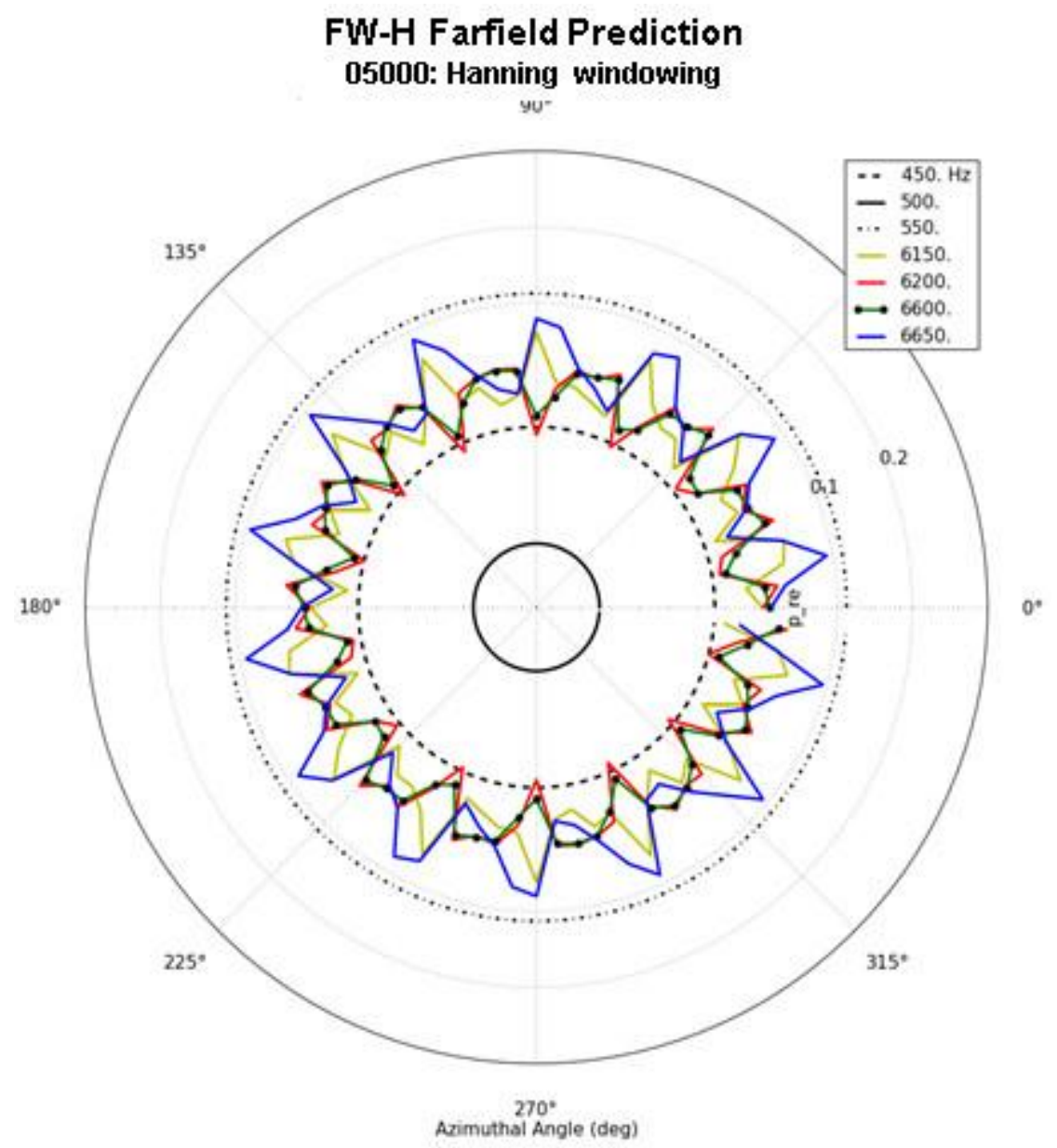

Figure 8. Azimuthal directivity patterns of selected narrow band frequencies.

\begin{tabular}{|c|c|c|c|c|}
\hline NPR $_{\text {prim }}$ & $\mathrm{NPR}_{\text {sec }}$ & NTR $_{\text {prim }}$ & NTR $_{\text {sec }}$ & H/D \\
\hline 1.157 & 1.168 & 2.588 & 1.056 & 0.213 \\
\hline
\end{tabular}

Table 1. Specifications re. operating condition and mixer lobe penetration height. 